\title{
A Case Report of Functional Acupuncture in the Treatment of Senile Left Persistent Facial Paralysis
}

\author{
Han Bingxue ${ }^{1}$, Shao Ming ${ }^{2}$, Liu Jing ${ }^{3}$, Sun Yu ${ }^{1}$, Cong Bei ${ }^{1}$, Du Fei ${ }^{1}$, Li Tong ${ }^{1}$, Yang Dan ${ }^{1}$, \\ Yan Zhi ${ }^{1,3}$, * \\ ${ }^{1}$ Department of Acupuncture and Tuina, Dalian Port Hospital, Dalian, China \\ ${ }^{2}$ Department of Neurology, the Affiliated Brain Hospital of Guangzhou Medical University, Guangzhou, China \\ ${ }^{3}$ Department of Neurology, Sichuan Rehabilitation Hospital, Chengdu, China
}

\section{Email address:}

yyr@dl.cn (Han Bingxue), 1961523453@qq.com (Shao Ming), 269807602@qq.com (Liu Jing), sunyu_bear@126.com (Sun Yu), Congpei@126.com (Cong Bei), dufei198813@126.com (Du Fei), hys907325@163.com (Li Tong), 525768434@qq.com (Yang Dan), qjqiaojie@126.com (Yan Zhi)

${ }^{*}$ Corresponding author

\section{To cite this article:}

Han Bingxue, Shao Ming, Liu Jing, Sun Yu, Cong Bei, Du Fei, Li Tong, Yang Dan, Yan Zhi. A Case Report of Functional Acupuncture in the Treatment of Senile Left Persistent Facial Paralysis. International Journal of Chinese Medicine. Vol. 5, No. 1, 2021, pp. 1-5. doi: 10.11648/j.ijcm.20210501.11

Received: January 17, 2021; Accepted: January 26, 2021; Published: February 9, 2021

\begin{abstract}
Introduction: Idiopathic facial palsy, also known as Bell's palsy (BP) or facial neuritis, is an acute onset of peripheral facial neuropathy. The onset of this disease is rapid, and there is a history of cold, mostly on one side of the face. Objective: to introduce and analyze the method and curative effect of functional acupuncture on one case of senile left persistent facial paralysis. Methods: focus on the functional parts of the affected area that need to be improved, select the acupuncture areas with improved trigger function, and select the stimulation dose of electroacupuncture. In other words, functional acupuncture is the main acupuncture method in the treatment process, that is, to take a functional position before acupuncture on the paralyzed side of the cheek, that is, to lift the patient's left corner of the mouth outward to above the functional position with a surgical small retractor (the largest lifting mouth angle is tilted to the left side), to determine the left cheek muscle functional area (needle entry area), and to make corresponding adjustment on the left cheek Acupoints selected in functional areas: Xiaguan, zygomatic Luo, buccal Che, Yingxiang, Renzhong, Taiyang, sizhukong, Chengzhi, yangbai. The needle of $0.35 \mathrm{~mm} \times 40 \mathrm{~mm}$ was selected, and $0.3-1$ inch flat needling was used with electroacupuncture. The waveform was density wave. Five times a week, 10 times for a course of treatment. Results: after two courses of functional acupuncture treatment, the patient's left eyelid was basically closed, and the air leakage during the left cheek bulging was significantly improved. After eating, the food left in the left cheek disappeared, the left nasolabial groove appeared, the left frontal lines appeared, and the symptoms of the left mouth angle slanting to the right were significantly improved (see the figure below). Conclusion: the essence of functional acupuncture improvement is to re form a strong neuromuscular effect in muscles, destroy the paralysis and weakness of target muscles, and reconstruct the normal neuromuscular coupling mode. The aim of this balance mechanism is to destroy the reflex pattern of abnormal muscle paralysis and muscle weakness and reconstruct an orderly and normal neuromuscular reflex.
\end{abstract}

Keywords: Elderly, Functional Acupuncture, Facial Paralysis

\section{Introduction}

Idiopathic facial palsy, also known as Bell's palsy (BP) or facial neuritis, is an acute onset of peripheral facial neuropathy. The onset of this disease is rapid, and there is a history of cold, mostly on one side of the face. It is common in winter and summer, and can occur at any age. The peak of the disease is after 40 years old, and the incidence is similar between men and women, with an annual incidence of $15-30 / 100,000$ [1]. Facial paralysis is not a rare disease, for 
example, the most common peripheral facial paralysis, Bell facial paralysis (about 2/3 of all patients with facial paralysis), has a incidence of about 20-30 people per 100,000 years, that is, 1 person in 60 people will suffer from this disease in their lifetime [2]. Facial nerve is one of the most circuitous cranial nerves, which perfectly interprets the complexity of cranial nerve anatomy and physiological function, and also makes the work related to facial nerve extremely attractive and challenging. Facial paralysis is to point to the facial neuropathy that causes as a result of various reasons and a series of corresponding clinical manifestations. Patients may present with facial deformation, disappearance of forehead lines, inability to close eyes, askew of the mouth, and leakage of the gills, accompanied by abnormal secretion of tears, speech disorders, changes in taste, etc., which may lead to serious consequences such as corneal ulcer and vision loss [3]. The left and right sides of the disease have the same incidence, among which a few are bilateral, accounting for $0.3 \% \sim 2 \%$ [4]. Facial paralysis will not only seriously affect the appearance of patients, but also have a great impact on their social behavior, work and life [5].

Among the grading methods reported in literature, House. Braekmann grading method, Burres. Fisch grading method and Toronto grading method are more widely used. Evans et al. [6] believe that House. Braekmann grading method is simple and reliable. But with few observers, the results were inconclusive. Smith et al. [7] also believe that House. Braekmarm grading method is the simplest and easiest to implement. The biggest drawback of this study lies in the lack of validity of the selected reference criteria. Yen, etc. [8] research shows that with the House. The brake. The manll classification method, an overall evaluation of facial paralysis on one side of the level and use the law to the brow, eyes, nose, mouth and other local evaluation of the level of coincidence rate is not high, in addition to the ocular coincidence rate was $61 \%$, the accuracy of other parts are under $40 \%$, shows that only with the overall evaluation is not enough to fully describe the facial features, which mainly reflects the eye function. Croxson et al. [9] conducted a comparative study of House. Brack. Nqann classification method and Burres. Fisch classification method, and found that there is excellent consistency between the classification results of the two methods, and that the reliability of House. Brackmann classification method is not high. Ross et al. [10] conducted a statistical test on the proposed TFGS, and the results showed that the three components were relatively independent of each other. There was no significant correlation between scores of any two parts, but scores of each part were equally significant correlated with comprehensive scores, thus proving the inherent effectiveness of the method itself.

\section{Case Description}

\subsection{History of Present Disease}

Patient Sun X, female, 67 years old, was admitted to the hospital at 13:14 on April 12, 2019 due to the right corner of the mouth skew for 7 hours. Chief complaint: on the day after morning rises found askew quarrel no incentive to the right, the left eyelid closure is not complete, the left eye tears, and the left cheek with food after eating, symptoms, no pain after ear, no taste, no hearing, no dizzy, head pain, no physical activity barriers, no nausea and vomiting, diplopia and vertigo attacks, today for the definite diagnosis and treatment to our hospital, outpatient service to my family, "facial paralysis" income no heart palpitations, chest pain, after onset of illness has no sense of abdominal distension, abdominal pain, normal diet, the mainland.

\subsection{Past History}

The patient had hypertension for more than 10 years, and had been taking "candesartan" at that time, and had not measured his blood pressure recently. The symptoms of palpitation and shortness of breath after normal activities were systematic examination, long-term use of "Aspirin" and "Salvia miltiorrhiza dripping pills", and intermittent use of "Kedine". He underwent lipoma resection on his left hand 18 years ago and 3 years ago, and underwent hemorrhoid surgery 8 years ago. He denied the history of intraoperative blood transfusion and suspected tuberculosis. Self-report of elevated transaminase after taking rosuvastatin. No history of diabetes, no history of hepatitis, tuberculosis and other infectious diseases, no history of trauma, blood transfusion, no history of drug and food allergy, vaccination is carried out with the society.

\subsection{Physical Examination}

$\mathrm{T}: 36.3 \mathrm{C}, \mathrm{P}: 70 / \mathrm{min}, \mathrm{R}: 18$ times/min, BP:138/86mmHg, low energy, clear breathing sound in both lungs, dry and wet rale sound was heard. Heart rate: 70/ min, Rhythm, P2, >, A2, abdomen soft, no tenderness, liver, spleen and costal, lower limbs no swelling. Nervous system: clear, left frontal lines become shallow, left eyelid inadequateness, positive Bell sign, left nasolabial fold becomes shallow, left corner of mouth is askew to the right, food remains on the left cheek after eating, air leakage when the left cheek is bulging, tongue extension is in the middle, muscle strength of the extremitiesis level 5, symmoxia is stable, bilateral Pap sign is negative (see Figure 1. After treatment.).

\subsection{Primary Diagnosis}

1) Left Persistent Facial Nerve Palsy

2) High Blood Pressure Level 3, Extremely High riSk Level

\subsection{Differential Diagnosis}

Brainstem infarction: the patient was an elderly female with a history of hypertension, but the patient had no abducens nerve injury and bilateral disorder, and the evidence of brainstem infarction was insufficient.

\subsection{Diagnosis and Treatment Plan}

1) Pay attention to rest, avoid blowing wind, catching cold, etc. 
2) Give symptomatic treatment to improve circulation and nourish the nerves

3) Improve liver function, kidney function, abdominal color ultrasound and other relevant examinations. The elderly patient was examined for carotid artery color ultrasound to determine whether there was arteriosclerosis and stenosis of the large extracranial arteries. The patient had a history of suspected tuberculosis, and lung CT was performed to confirm.

4) Disclosure of illness may have sequelae.

\subsection{Admission Examination Results}

1) Multiple miliary nodules in the upper lobe of the left lung and the lower lobe of both lungs, followed up;

2) Aorta and left coronary artery sclerosis.

3) Head MRI: a) Demyelination of brain proteins; b) Left anterior cerebral artery A1 segment absent.

4) The virological results of liver function were normal.

5) Blood routine: white blood cell count WBC11.17-109/L $\uparrow$, neutrophils absolute value NEUT\# 8.36 109/L. Routine urine results were normal. The result of routine stool is normal.

6) Tumor markers (AFP, CEAP, CA125P, CA199P, CA153) were normal.

7) The liver and kidney function results were normal.

8) Abdominal ultrasonography: liver, gallbladder, spleen, pancreas and kidneys were not abnormal.

9) Electrocardiogram: sinus rhythm, generally normal electrocardiogram.

10) Carotid ultrasound:

a) Local thickening of endomedia at the right carotid artery bifurcation

b) Plaque formation at bilateral carotid artery bifurcation. On admission, the diagnosis was clear and symptomatic treatment was given, such as improving circulation, nourishing nerves, acupuncture and moxibustion.

\section{Methods and Results}

After 3 months of circulation improvement, nerve nutrition and conventional acupuncture treatment, there was no significant improvement. After joint consultation, the treatment was performed according to the functional acupuncture method developed by Professor Yan Zhi:

\subsection{Functional Acupuncture Therapy}

Prior to the needle into patients left quarrel with surgical retractor to function or to the left of the skew, auxiliary force closed eyelids, determine the left face and around his left eye into the pin function, for patients with facial paralysis we selected points are: shimonoseki, zygomatic liao, buccal car, yingxiang, among, the sun, te 23, pulp, Yang bai, choose the type of $0.35 \mathrm{~mm} \times 40 \mathrm{~mm}$ needle, take $0.3 \sim 1$ inch flat spines, cooperate with electric acupuncture, waveform as the density wave, five times a week, 10 times for a period of treatment.

\subsection{For Cheek Muscle Training}

First lift the patient's left mouth Angle to the functional position or the left side askew position with a surgical retractor, and then conduct the left side mastication training with 30 pieces in each group, 4-6 groups each time, 4-6 times a day.

\subsection{Four Kinds of Rehabilitation Training for Eyelid Incompleteness}

\subsubsection{Eye Closed Exercise}

The doctor expands the muscles around the patient's eyelids up and down by hand, forming the maximum open state of the eyes, and then tells the patient to try his best to close his eyes, forming the anti-resistance closing action of the eye racket muscle. Each time the anti-resistance closing action is $10-15$ seconds, and then the patient rests for 5 seconds. Each group is repeated 5-8 times, 4-6 groups/day.

\subsubsection{Rainfall Distribution on 10-12. Massage}

Use the abdomen of the ring finger of both hands to press the eyebrow and the center of the eyebrow close to the upper eyelid until you feel local soreness. Repeat 5 to 8 times a day, 4 to 6 times a day.

\subsection{The Results}

After 2 courses (20 days) of functional acupuncture based comprehensive treatment, the patient's left eyelid was basically closed, the left cheek leakage was significantly improved, the left cheek food phenomenon disappeared after eating, the left nasolabial groove appeared, the left frontal lines appeared, and the left mouth Angle to the right was significantly improved (see Figure 2. After treatment.).

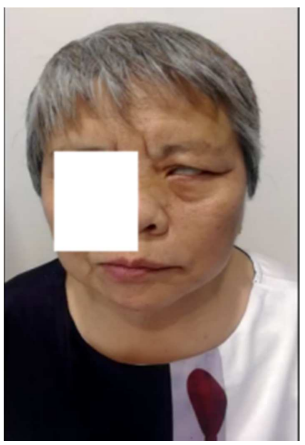

Figure 1. Before treatment.

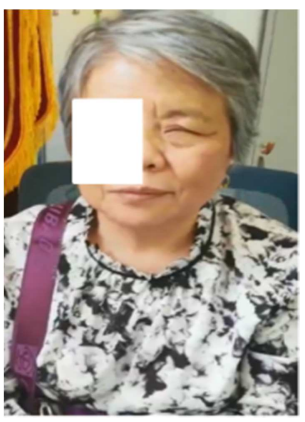

Figure 2. After treatment. 


\section{Discussion and Conclusion}

Electrophysiological examination of facial nerve disease include: dc test, nerve excitement test, stimulation test, the largest neural electrical diagram (Electroneuronography, ENoG), electromyography (EMG), blink (BR) and F wave, etc. The first three types of tests are being used less and less because they rely on operator observation or subject subjective feeling. Enog and EMG are currently widely used in clinical practice [11]. Enog recorded Compound Muscle Action Potentials (CMAPS) at the corresponding region of the facial region during electrical stimulation of the main facial nerve after the stem and mammaplasty foramen. If the neuropathy has not involved the distal end of the facial nerve behind the mammary foramen of the stem, the results of eNOG can be normal, and the process lasts about 72 hours [12, 13]. After that, the degree of nerve degeneration can be estimated by comparing the amplitude difference of bilateral CMAPS when the neurodegeneration progresses to the distal nerve without significant atrophy of facial muscle fibers [14]. When the neural degeneration exceeded $53.5 \%$, the possibility of linkage was significantly increased [15]. Due to the coexistence of nerve degeneration and regeneration after 3-4 weeks of facial paralysis, atrophy of muscle fibers and formation of nerve bypass, the monitoring value of electronerography for facial paralysis is greatly reduced, and its measurement results lag behind clinical recovery or progress [16].

Facial paralysis can cause many changes in social cognition, especially changes in the whole body and the middle part of the face [17]. The evaluation of social function after facial paralysis and the patient's self-assessment are also important aspects of bedside assessment. At present, there are as many as 28 questionnaires involving facial paralysis, but only 3 scales are more suitable for self-assessment of life quality after facial paralysis, and the evaluation of facial features and facial motor symptoms of these scales is not satisfactory [18]. Facial Clinimetric Evaluation Scale is the most widely used self-assessment Scale, with a total of 15 items. After cluster analysis, it is found that Facial local movements, Facial sensation, social influence, psychological activities, chewing function and eye influence are mainly included. There was a moderate correlation between items related to facial local activity and the Sunnybrook grading system.

The core of functional acupuncture method is to weaken and improve the traditional meridian and acupoint acupuncture, and on the premise of ensuring safety, the targeted muscles that can immediately or obviously stop symptoms (mostly the muscles that cause paralysis as the target) are selected as the target of acupuncture. The essence of this function improvement is that the muscles re-form a powerful neuromuscular effect, destroy the paralysis of targeted muscle groups, and rebuild the normal neuromuscular coupling mode. The goal of this balancing mechanism is to re-regulate the higher regulation center of the brain. With functions of acupuncture method of destroying abnormal myoparalysis and reflection patterns of muscle weakness, reconstruction and orderly, normal neuromuscular reflex, increase the acupuncture in the treatment of dynamic facial nerve paralysis caused by eye skewed method of weight, through quickly relieve symptoms help patients overcome before and anxiety in the treatment, to achieve the effect of homology of body and mind. Needle punching operation emphasize function position (that is, we want to achieve the effect of the treatment), the function of the patients posture is passive pull on the left side of the mouth to normal or skewed to the left, the purpose is to make the left side of the muscles in the state of relaxation, and then select the shimonoseki, zygomatic liao, buccal car, yingxiang, among, the sun, te 23, pulp, Yang bai, save bamboo, fish waist, te 23 , o god court, head Lin chi and is hole as the needle point, such a straight acupuncture methods, are more likely to make pulled loose targeted muscle contraction, fast with needle function under a same function after training, the treatment effect is significantly faster. Functional acupuncture attaches great importance to the improvement of local functions of patients, and emphasizes innovation and promotion in the process of inheriting traditional Chinese medicine. It strives to make functional acupuncture become the safest, most stable and best therapeutic technique in the intervention of facial neuritis, and makes efforts to innovate and enrich the application value and evidence chain of acupuncture.

\section{Acknowledgements}

This work was supported by the Natural Science Foundation Guidance Project of Liaoning Province, China (Grants No. 20180550180; 20180550069; 2019-ZD-1000; 2019-ZD-1002).

Science and Technology Planning Project of Sichuan Province, China (Grants No. 2018JY0664).

\section{References}

[1] Neurology Society of Chinese Medical Association, Neuromuscular Group of Neurology Society of Chinese Medical Association, Electromyography and Clinical Neurophysiology Group of Neurology Society of Chinese Medical Association. Clinical Guidelines for Diagnosis and Treatment of Idiopathic Facial Palsy in China [J]. Chinese Journal of Neurology, 2016, (2). 84-86.

[2] De Almeida J R, Guyatt G H, Sud S, et al. Management of Bell palsy: clinical practice guideline [J]. CMAJ: Canadian Medical Association journal $=$ journal de l'Association medicale canadienne, 2014, 186 (12): 917-922.

[3] KONG Yan, XU Zhuo, HAO Yanan, et al. Evaluation of facial nerve function scale in idiopathic facial palsy $[\mathrm{J}]$. Gao ZQ. Development of methods for evaluating facial nerve function [J]. Chinese Journal of Ocular Science, 2019, 17 (4). 445-451.

[4] Wang J, Ling L. Research progress on etiology and pathogenesis of Bell's palsy [J]. Chinese Journal of Neuroimmunology and Neurology, 2013, (2). 138-140.

[5] Macgregor F C. Facial disfigurement: problems and management of social interaction and implications for mental health [J]. Aesthetic plastic surgery, 1990, 14 (4): 249-257. 
[6] Evans RA, Harries NL, Bagulry DM, et a1. ReliabilityoftheHouseand Brackmann gradingsystem forfacialpalsy. JLaryngolOto1. 1989; 103: 1O45-1046.

[7] Smith IN, Murray JAN, Cull RE, et a1. A compari offacialgrading systems. Clin Otolaryngo1. 1992: 17: 303-307.

[8] Yen TL; Driscoll CL; Lalwani AK. Significance of House-Brackmann facial nerve grading global sccren in the setting of diferential facial nerve function. Otol Neuroto 1. 2003; $24: 118-122$.

[9] Croxson G. Mayl, Mester SJ. Grading facial nerve function: House-Brackman versus Burres-Fisch methods. Am J Oto1. 1990; 11: 240-246.

[10] Ross BG. Fradet G. Nedzelski JM. Development of asensitiveclinical facialgrading system.

[11] Otolaryngol Head Neck surg. 1996; 114: 380-386.

[12] Baugh R F, Basura G J, Ishii L E, et al. Clinical practice guideline: Bell's palsy [J]. Otolaryngology-head and neck surgery: official journal of American Academy of Otolaryngology-Head and Neck Surgery, 2013, 149 (3 Suppl): S1-27.

[13] Lee D H. Clinical Efficacy of Electroneurography in Acute Facial Paralysis [J]. J Audiol Otol, 2016, 20 (1): 8-12.
[14] Ozgur A, Semai B, Hidir U U, et al. Which electrophysiological measure is appropriate in predicting prognosis of facial paralysis? [J]. Clinical neurology and neurosurgery, 2010, 112 (10): 844-848.

[15] Mannarelli G, Griffin G R, Kileny P, et al. Electrophysiological measures in facial paresis and paralysis $[\mathrm{J}]$. Operative Techniques in Otolaryngology-Head and Neck Surgery, 2012, 23 (4): 236-247. Azuma T, Nakamura K, Takahashi M, et al. Electroneurography in the acute stage of facial palsy as a predictive factor for the development of facial synkinesis sequela [J]. Auris Nasus Larynx, 2018, 45 (4): 728-731.

[16] Arslan H H, Satar B, Yildizoglu U, et al. Validity of late-term electroneurography in Bell's palsy [J]. Otology \& neurotology: official publication of the American Otological Society, American Neurotology Society [and] European Academy of Otology and Neurotology, 2014, 35 (4): 656-661.

[17] Li M K, Niles N, Gore S, et al. Social perception of morbidity in facial nerve paralysis [J]. Head Neck, 2016, 38 (8): 1158-1163.

[18] Ho A L, Scott A M, Klassen A F, et al. Measuring quality of life and patient satisfaction in facial paralysis patients: a systematic review of patient-reported outcome measures [J]. Plast Reconstr Surg, 2012, 130 (1): 91-99. 